\title{
REGION OF INTEREST BASED COMPRESSION OF MEDICAL IMAGES USING VECTOR QUANTIZATION
}

\author{
M.Mary Shanthi Rani and P.Chitra \\ Department of Computer Science and Applications, Gandhigram Rural University, \\ Gandhigram,Dindigul,Tamil Nadu, India
}

\begin{abstract}
In this paper, a Region of Interest(ROI) based compression method is proposed using Vector Quantization $(V Q)$ to enhance the image quality for efficient transmission and storage. Vector quantization is an effective lossy compression method to compress an image with good quality.. Telemedicine, which is gaining more significance recently, requires massive data transmission between various locations in short duration. The proposed method mainly focuses on compressing medical images with different codebook sizes for region of interest and non region of interest using vector quantization method.The proposed method achieves high compression ratio without compromising the quality of reconstructed ROI image.
\end{abstract}

\section{KEYWORDS}

Image Compression, Vector Quantization, K-Means Clustering, ROI Compression

\section{INTRODUCTION}

The recent era technology requires online processing for each and every application which demands high speed transmission with reduced storage space. Compression satisfies the requirements of data transmission without degrading the quality with minimum storage. Especially medical image data occupies huge amount of space. Image compression plays a vital part in image processing techniques. The process of compression is to reduce the data size without degradation of the original data. The basic operation of compression is to reduce the redundancy data from the original data.

Basic data redundancies are

1. Coding Redundancy

2. Inter-Pixel Redundancy

3. Psycho-visual Redundancy

The Scheme of Image Compression aimed to reduce the transmission cost for images, while maintaining a good level of visual quality[1]. Research aspect of compression can be applied for the demands of fast transmission of medical images. Various methods have been proposed for image compression such as Transforms, Vector Quantization, evolutionary computation techniques etc.

Image compression can be categorized into Lossy and Lossless compression techniques. The original image can be perfectly recovered from the compressed image, in lossless compression

DOI :10.5121/ijcsity.2016.4103 
techniques. This technique is widely used in medical field for compressing enormous data with high quality[2].The impact of a lossless compression is getting a high computational complexity.

Some of the popular Lossless compression techniques are

-Run-Length Coding

-Huffman Coding

-LZW Coding

-Arithmetic Coding

Lossy compression methods reduce redundant information from the original data with low computational complexity. The lossy compression that produces slight differences may be considered as visually lossless. This is the challenge for achieving the quality of medical image data, which is mainly used in still image transmission. Lossy methods are used for most applications. The reconstructed image is not a exact copy of an original image but it can resemble the original image[2].Common lossy compression techniques are

- Block Truncation Coding

- Vector Quantization Coding

- Transform Coding

- Fractal coding

\subsection{Vector Quantization Algorithm}

Vector Quantization(VQ) is one of the most powerful lossy compression method that produces good quality images with low computation complexity.VQ has enormous applications in the fields of image and video compression, Pattern Recognition, and Watermarking, However, it has some complexity depending on choosing number of clusters and random selection of initial seeds. Wrong choice of initial seeds may yield poor outcome and results in increase in time for convergence[3].

A VQ has two operations such as encoder and decoder. The encoder compare search input vector with every code vector in the codebook and generate index of the code vector which represent the minimum difference from the input vector. A decoder takes the indexes to locate the code vector in codebook and generate the output vectors[4].

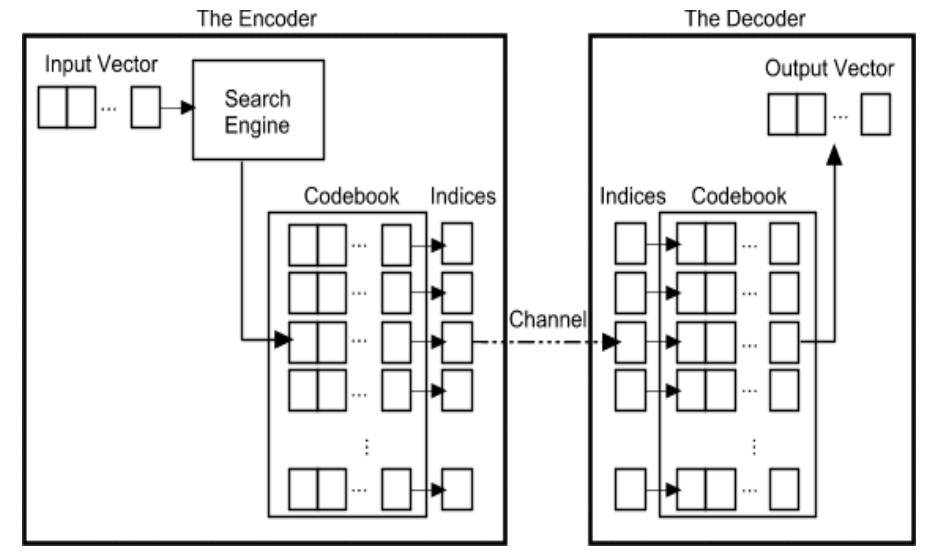

Figure 1: Process of VQ 
VQ includes various stages in their process: Formation of the Vector, Creation of Training set vectors, Generation of the codebook and finally quantization of input image data.

\section{Algorithmic Steps:}

Step 1: Divide the input image into non overlapping blocks and convert each block into vectors.

Step 2: Randomly generate an initial codebook CB0

Step 3: Initialize I $=0$.

Step 4: Perform the following process for each training vector.

- Compute the distance between all the training vectors belonging to this cluster and the codewords in CBI.

- Compute the centroid (code vector) for the clusters obtained in the above step.

Step 5: Increment I by one and repeat the step 4 for each code vector.

Step 6: Repeat the Step 3 to Step 5 till codebook of desire size is obtained.

Vector quantization (VQ) algorithms that employ an pioneering approach to VQ using number patterns as the codebook have been proposed which outperforms JPEG in producing high quality images [5-6].

\subsection{K-Means Clustering Algorithm}

$\mathrm{K}$-means algorithm is widely used in VQ technique known for its efficiency and speed. This is a widespread iterative clustering algorithm that generates anoptimal codebook which is a setof cluster centroids from the training data using an appropriate distance function suitable for the given application.

The main factor that determines the performance of K-means is its convergence time which mostly depends on the amount of training data, codebook size, code vector dimension, and distortion measure [7].The overall quality of clustering is the average distance from each data point to its associated cluster centroid.

The efficiency of K-means algorithm depends upon the initial selection of seeds. Hence many novel methods have been proposed for selecting the initial seeds for K-means Clustering[8-9]. Two new methods have been proposed in [10-11] which select the initial seeds based on proper blend of statistical parameters mean, median and mode.

In this paper, a new method has been proposed for finding the initial seeds of K-means clustering using Vector Quantization. VQ is based on K-means Clustering technique. 
The Basic algorithm for K-Means Clustering is listed below:

1: Select $\mathrm{K}$ random points as the Initial centroids

2: Repeat Steps 3-4

3: From $\mathrm{K}$ clusters by assigning all points to the closest centroid

4: Recomputed the centroid of each cluster

5: Until the centroids don't change

\subsection{Region of Interest (ROI)}

ROI is commonly used in medical imaging to diagnose animportant region. The purpose of ROI is used to identify or measuring the image portions of interest. Region of Interest is selecting the regions according forthe user choice. Compress the ROI with the image will be compressed by both lossless and lossy compression techniques. After decompression, the images will be compared with the results andby different imagequality measures.Main goal of Region of interest(ROI) compression is to compress ROI with high quality as compared to the Background. For an example, while compressing medical image the diagnostically important region should be compressed with better quality than background. Thus, the ROI area is compressed with less compression ratio and the background with the highest possible compression ratio in order to get overall better compression performance[12]

The rest of the paper is organized as follows: Section 2 describes the proposed method along with the flow diagram, Section 3 presents the results and discussion of the performance of the proposed method and Section 4 concludes with the future enhancements.

\section{PROPOSED METHOD}

In this paper a Lossy image compression method using vector quantization technique for compressing image has been proposed. It can achieve a quality image with high resolution. The proposed method has three phases.

The first phase starts with segmenting an image into three components such as Image1, Image2 and Region of Interest (ROI).In this form Image 1 and Image 2 represents the Background of the Image. Region of Interest is the vital part of the image crucial for medical diagnosis. Vector quantization is applied to these three components with different block sizes. Codebooks are generated for Image1 and Image 2 with $8 \times 8$ as a block size. Further to get quality image of ROI, $2 \times 2$ block size is chosen. For applying VQ to enhance the quality[13-14] of the codebook, initial seeds are generated by choosing the blocks with high variance. This process leads towards simple computation and less complexity.

\subsection{Compression Procedure of Image1 and Image2}

Step1: Divide Image1 and Image2 into $8 \times 8$ blocks, which is co nsider as a training set of vectors.

Step2: Apply K-means clustering and generate the Codebook, which is the set of cluster centroids separately for Image1 and Image2. 


\subsection{Compression Procedure of ROI region}

Step1: Divide ROI image into 2x2 blocks to form the ROI training set.

Step2: Choose blocks of high variance as the initial seeds

Step3: Apply K-means clustering to the ROI training set using the initial seeds in step2.

\subsection{Proposed Flow Diagram}

The proposed method process is pictorially represented in the following flow diagram.

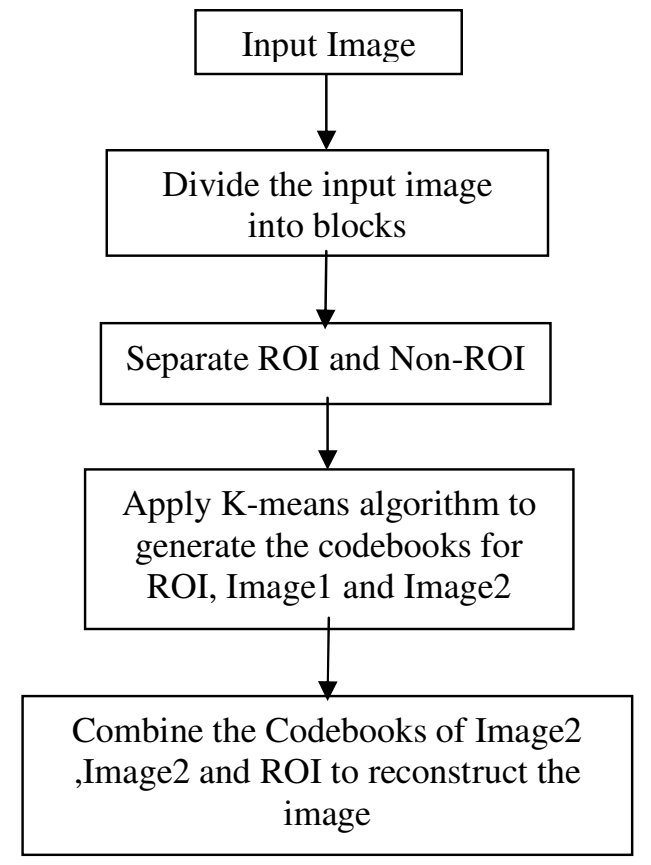

Figure 2. Flow Diagram of the proposed method

The above flow diagram Figure 2 explains the operation of the entire process. This shows the short description about the proposed work.

\section{RESULTS AND DISCUSSION}

Experiments are conducted on four different images with different block sizes $8 \times 8$ for training set vectors of Non-ROI region and $2 \times 2$ block size for ROI region. The results reveal that the proposed method yields better quality image than the K-means random seeds selection method in terms of picture quality, which is the crucial factor for accurate medical diagnosis.

The performance of the proposed method is assessed based on the metrics MSE, PSNR ,CR and CT.

The Mean Square Error (MSE) and the Peak Signal to Noise Ratio (PSNR) are used to compare image compression quality.

1.Mean Square Error (MSE) 
The MSE represents the cumulative squared error between the compressed image $y_{c}$ and the original image $y_{0}$. The lower the value of MSE, the lower the error.

$$
M S E=\frac{\Sigma\left(y_{c}-y_{0}\right)}{n}
$$

\section{Peak Signal to Noise Ratio (PSNR)}

PSNR is often used as a quality measurement between the original and the compressed image, measured in decibels. This is computed using the following equation:

$$
P S N R=20 \log _{10} \frac{255}{\sqrt{\text { MSE }}}
$$

The higher value of the PSNR, the better and the quality of the compressed or reconstructed image.

\section{Compression Ratio (CR)}

Compression Ratio finds the compressed range of the reconstructed image, which defines the quality of an image.Computation time shows the effect of proposed method.

$$
\text { Compression Rulio }=\frac{\text { size of the originaifile }}{\text { size of the comprased fils }} \text { (3) }
$$

The performance comparison of the proposed method and Random K-means with random initialization of seeds are presented in Table 1 . The experimental results demonstrate that the proposed method produces good quality images in less computation time when compared with K-means with random seeds.

Table 1. Result of Proposed Method

\begin{tabular}{|c|c|c|c|c|c|}
\hline $\begin{array}{c}\text { Image } \\
\text { Type }\end{array}$ & Method & MSE & PSNR & $\begin{array}{c}\text { Computation } \\
\text { Time } \\
\text { (In Sec) }\end{array}$ & $\begin{array}{c}\text { Compressi } \\
\text { on Ratio } \\
(\%)\end{array}$ \\
\hline MRI Brain & K-means & 93.385 & 28.462 & 19.867 & 28.46 \\
\hline & Proposed Method & 81.684 & 29.043 & 4.149 & 120.47 \\
\hline Iris Image & K-means & 104.096 & 27.990 & 7.792 & 17.34 \\
\hline & Proposed Method & 82.028 & 29.025 & 2.764 & 61.92 \\
\hline $\begin{array}{c}\text { Mammogra } \\
\text { m }\end{array}$ & K-means & 35.947 & 32.608 & 10.681 & 15.36 \\
\hline & Proposed Method & 55.991 & 30.684 & 3.728 & 67.76 \\
\hline PET Image & K-means & 55.240 & 30.742 & 18.743 & 27.36 \\
\hline & Proposed Method & 58.356 & 30.504 & 4.653 & 97.52 \\
\hline
\end{tabular}


The original and reconstructed images of MRI_Brain, Iris, Mammogram and PET using the proposed method and K-Means have been shown in Fig.1, Fig.2, Fig.3 and Fig.4 respectively.
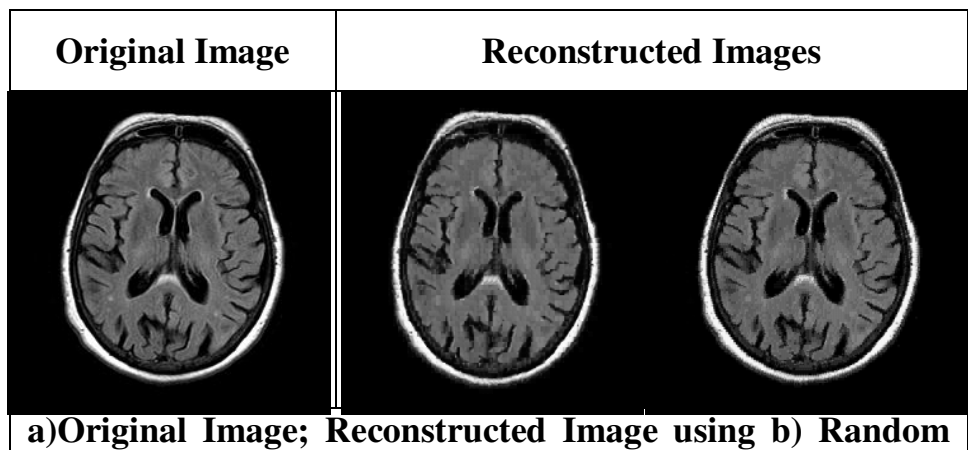
K-means c) Proposed Method

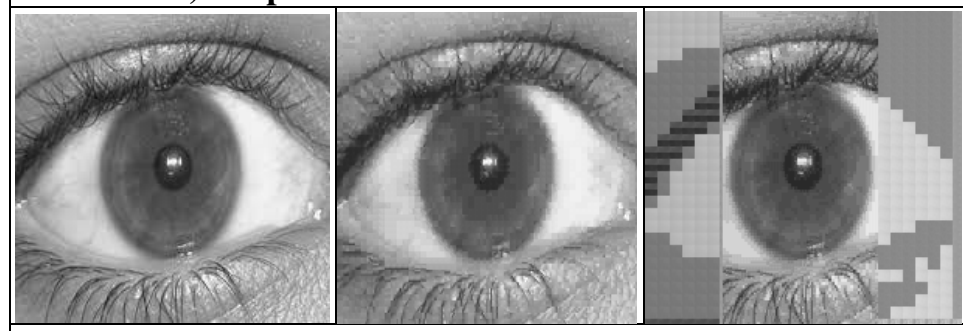

a)Original Image; Reconstructed Image using b) Random K-means c) Proposed Method

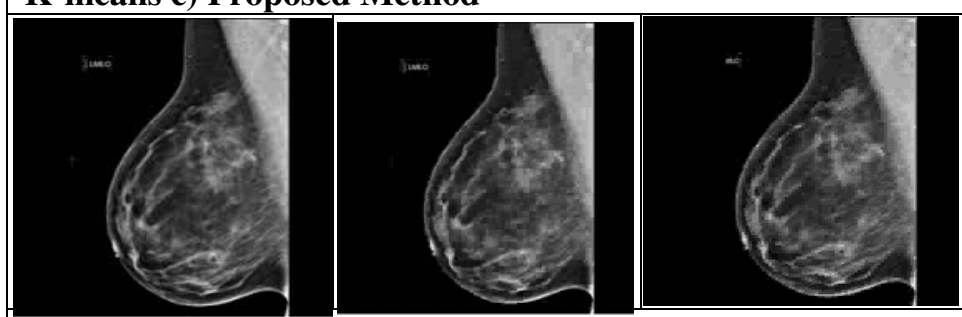

a)Original Image; Reconstructed Image using b) Random K-means c) Proposed Method

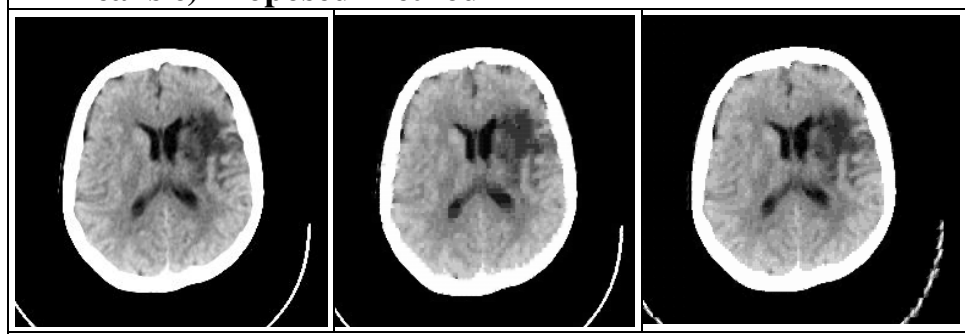

a)Original Image; Reconstructed Image uisng b) Random K-means c) Proposed Method 


\section{CONCLUSION}

This paper presents a ROI based image compression technique for compressing medical images and the experimental results show that this will be an ideal choice for telemedicine applications. Our future work will be enhancing the performance further by applying Residual Vector Quantization.

\section{REFERENCES}

[1] G.M.Padmaja, P.Nirupama "Analysis of Various Image Compression Techniques", ARPN Journal of Science and Technology, ISSN 2225-7217 VOL. 2, NO. 4, May 2012.[2] Athira B.Kaimal, S. Manimurugan, C.S.C.Devadass, "Image Compression Techniques: A Survey", International Journal of Engineering Inventions e-ISSN: 2278-7461, p-ISBN:/2319-6491 Volume 2, Issue 4 , PP: 2628,February 2013.

[3] KishorAtkotiya , ChetanDudhagara, "Image Compression using VectorQuantization", International Journal of IT, Engineering and Applied Sciences Research (IJIEASR) ISSN: 2319-4413 Volume 2, No. 2, February 2013

[4] K.Somsundaram, Mary Shanthi Rani, Adaptive Classified Pattern Matching Vector Quantization Approach for compressing images, The 2009 Internationa Conference on Image Processing, Computer Vision \& Pattern Recognition Proceedings, Las Vegas, USA., pp.532-538, July 2009

[5] K.Somsundaram, Mary Shanthi Rani, A Pattern Based Residual Vector Quantization algorithm for compressing images, International conference on Current Trends in Information Technology Proceedings, IEEE Computer Society, Dubai,pp.26-32, December 2009.

[6] M.MaryShanthi Rani, "A Genetic Algorithm Based K-Means Algorithm For Compressing Images", International Journal Of Engineering And Computer Science ISSN: 2319-7242 Volume 4, Page No. 14359-14362, Issue 9 Sep 2015.

[7] M.Mary Shanthi Rani, "Residual Vector Quantization Based Iris Image Compression", International Journal of Computational Intelligence Studies, Vol.3, No.4, pp.329-334,Inderscience Publishers, 2014

[8] K.Somasundaram, M.MaryShanthi Rani, "Mode Based K-Means Algorithm with ResidualVector Quantization for Compressing Images" Springer Berlin Heidelberg, 2011.

[9] K.Somasundaram, M.MaryShanthi Rani," Eigen Value based K-means Clustering for Image Compression", International Journal of Applied Information Systems (IJAIS) - ISSN : 2249-086 Foundation of Computer Science FCS, New York, USA Volume 3- No.7, August 2012

[10] K.Somasundaram,M.MaryShanthi Rani, "Novel K-means algorithm for compressing images, International Journal of Computer Applications, Vol.18, No.8, pp. 9-13, ISSN 0975-8887, March 2011

[11] Venkateswaran, N. and Y.V. RamanaRao, "K-Means Clustering Based Image Compression inWaveletDomain",Information Technology Journal , Vol. 6, pp.148-153, 2007

[12] PratikChavada, Narendra Patel, KanuPatel, "Region of Interest Based Image Compression", International Journal of Innovative Research in Computer and Communication Engineering, Vol. 2, Issue 1, January 2014

[13] Mary ShanthiRani ,"A Hybrid Clustering Method for Compressing Iris Images", International Journal of Current Research, Vol 5,Issue 12, pp.3966-3969,December 2013

[14] Mary Shanthi Rani, Novel Residual Vector Quantization Method For Compressing Color Images, International Research Journal of Signal Processing, Vol 04, Article 057,pp.158-165, May 2013

\section{ACKNOWLEDGEMENTS}

I would like to express my heartfelt thank my supervisor Dr.M.MaryShanthi Rani. She has supported me in all direction under research of my topic. She has put a lot of effort and give an idea for the preparation of this paper. 


\section{Authors}

Dr.M. Mary Shanthi Rani,a NET qualified Assistant Professor in the Department of Computer Science and Applications, Gandhigram Rural Institute (Deemed University), Gandhigram hastwelve years of teaching and eight years of research experience as well. She has nearly twenty publications in International Journals and Conferences.Her research areas of interest are Image Compression, Information Security,Ontology, Biometrics and Computational Biology.She has authored a book titled "Novel Image Compression Methods Based on Vector

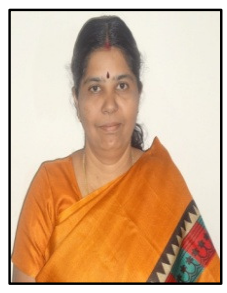
Quantization" and is one of the editors of Conference Proceedings "Recent Advances in Computer Science and Applications". She has served in various academic committees in designing the curriculum for B.Sc. and M.C.A courses as well. She has also served as reviewerof Peer-reviewedInternationalJournals and Conferences and is a Life member of Indian Society for Technical Education. She has the credit of being the Associate Project Director of UGC Indo-US $21^{\text {st }}$ Knowledge Initiative Project.

P.Chitra has pursuing Ph.D in Computer Science in Gandhigram Rural University. She has received M.Phil Degree during 2013.Her research area of interest is Image Compression. She has published papers for National conference on "Recent Advances in Computer Science and Applications" on 19th and 20th March 2015 and National conference on "New Horizons in Computational Intelligence and Information System (NCNHCIIS-2015)" on 17th and 18th December 2015 in Gandhigram Rural Institute- Deemed university.

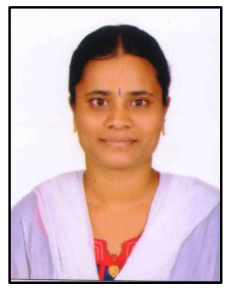

